\title{
HLA-Antigene bei germinatiyen Tumoren von verschiedener Lokalisierung
}

\begin{tabular}{|l|l|l|}
\hline J. & Abráhamová \\
\hline A. & Májský \\
\hline J. & Koutecký \\
\hline
\end{tabular}

Onkologische Klinik, Medizinische Fakultät, Karls-Universität, Prag Institut für Hämatologie und Bluttransfusion, Prag Department für Kinderonkologie, Pädiatrische Fakultät, Prag

\section{Schlüsselwörter}

Germinative Geschwülste

HLA-Antigene

Zusammenfassung

Bei 41 Kindern mit germinativen Tumoren von verschiedener Lokalisierung und 301 gesunden Kontrollpersonen wurden an Lymphozyten 22 HLA-Antigene des Lokus A und B typisiert. Kein statistisch signifikanter Unterschied in der Frequenz - mit Ausnahme der höheren ,Häufigkeit von HLA-A28 (14,63 \% gegenüber 4,32 \% bei Kontrolle, $\chi^{2}=$ $5,69)$ vor der Durchführung der Korrektur des p-Wertes -wurde gefunden. Die Ursache der selten existierenden Beziehungen zwischen den Geschwülsten und HLA-Antigenen wurde diskutiert.

MUDr. J. Abrahámová, Onkologische Klinik, U nemocnice 2, 12808-Praha, CSSR

Summary and Key Words

In 41 children with germ-cell tumors differently localised and 301 healty unrelated subjects, 22 HLA antigens of A and B loci were tested. No statistically significant difference in the frequency of HLA antigens - with exception of a higher frequency of HLA-A28 (14,63\% versus 4,32 \% in the controls, X2 = 5,69) before the correction of p value - was found. The cause of the rare associations between the tumors and HLA was discussed.

Germinative tumors- HLA antigens

\section{Einleitung}

In letzter Zeit wurde die Aufmerksamkeit der klinischen und theoretischen Wissenschaftler den germinativen Tumoren zu-gewendet. Ihre Diagnostik wurde verbessert; kombinierte Therapie führte zu einer wesentlich besseren Prognose. Die germinativen Geschwülste stellen eine sehr bunte Gruppe dar, zu der folgende Tumoren gehören: Seminom, embryona-les Karzinom, Dysgerminom, Teratokarzinom, Chorioepithe-liom und reifes Teratom. Alle genannten Geschwülste zeigen sich als bösartig - mit Ausnahme des reifen Teratoms, das für eine gutartige Erkrankung gehalten wird. Man nimmt an, daß sich wenigstens ein Teil der germinativen Geschwülste schon in der frühzeitigen Embryogenese ent-wickelt: Primordielle germinative Zellen wandern aus dem Entoderm des hinteren Abschnittes des Dottersacks in das Gebiet der urogenitalen Crista und bilden dadurch die notwendigen Bedingungen für das Auftreten von germinativen Geschwülsten [6]. Pierce und Dixon [12] und ferner Stevens [13] wiesen nach, daß alle germinativen Tumore aus einer omnipotenten Urzelle stammen. Für die Wahrscheinlichkeit 
der Existenz der genetischen Faktoren in der Pathogenese der germinativen Geschwülste sprechen zwei Umstände: 1.) Das von manchen Autoren festgestellte Familienvorkommen aller Typen der germinativen Tumore. 2.) Das beiderseitige Auftreten einiger Tumore (z. B. des Seminomes oder der Ovarialtumore). Genetische Faktoren wurden auch bei anderen Geschwülsten, wie beim Retinoblastom, dem Wilms’Tumore und dem Neuroblastom [8] nachgewiesen. Beim Retinoblastom [3] und Wilm's Tumor [1, 11] wurde eine Beziehung zum HLA-System festgestellt. Demgegenüber wurde kein Zusammenhang zwischen den Seminomen und Nicht-Seminomen (Teratokarzinome, embryonale Teratome) und dem HLA-System gefunden $[10,16]$.

Material und Methode

Patientengut: 41 Kinder mit germinativen Tumoren von verschiedener Lokalisierung, daraus 28 Kinder mit benignen Teratomen (10 Fälle mit sacrococygealen Teratomen, 2 mit retroperitonealen Teratomen, 1 Fall mit Mediastinumteratom, 5 Kinder mit Hodenteratom, 10 mit Ovarialteratom). 13 Kinder mit malignen germinativen Geschwülsten (8mal Ovarialdysgerminom, 4mal bösartiges Hodenembryom, Imal sacro-coccygeales Embryom).

Kontrollgruppe: 301 gesunde nichtverwandte Menschen. Untersuchungsmethode:

Mikrolymphozytotoxischer Test im Zweiphasenverf ahren NIH.

Untersuchungen: An Lymphozyten der 41 Kranken und 301 Kontrollpersonen wurden 22 HLAAntigene des Lokus A und B [Al, 2, 3, 9, 10, 11, 28, Awl9; B5, 7, 8, 12, 13, 14, 15, 17, 18, 27, 40, Bw21, 22, 35] bestimmt und ihre Frequenz in beiden Gruppen ausgerechnet.

Statistische Bewertung: Die Häufigkeit der HLA-Antigene in beiden Gruppen (bei Kranken und Gesunden) wurde mit Hilfe des X 2-Tests mit Yatesscher Korrektur, bzw. mit Woolf-Test im Falle der niedrigen Zahlen der Patienten statistisch verglichen. Die errechneten p-Werte wurden durch Multipli-zieren mit 22 korrigiert.

Ergebnisse

Die unterschiedlichen Frequenzen von HLA-Antigenen bei benignen Teratomen gegenüber der Kontrolle waren sämtlich statistisch nichtsignifikant. Dies gilt sowohl für höhere Häufigkeit einiger HLA-Antigene [All, A28, B7, Bw22], als auch für niedrigeres Vorkommen [A2, Aw19, B5, B12] und Abwe-

20

Abrahamova et al.: HLA-Antigene und germinative Tumore

Tabelle I. Frequenz von 22 HLA-Antigenen bei germinativenTumoren HLA- Germinative Tumoren Insgesamt Gesunde

$\begin{array}{lllll}\text { Antigen } & \text { Benigne } & \text { Maligne } & (\mathrm{n}=41) & (\mathrm{n}=301) \\ \begin{array}{l}\text { Teratome Tumoren \% } \\ (\mathrm{n}=28)\end{array} & \% & & \\ \% \quad \% & & & \end{array}$

senheit anderer HLA-Antigene bei Kranken [B14, B17, B18, B27, Bw21]. (Tabelle I). Die Abweichungen bei malignen germinativen Geschwülsten waren ebenfalls nicht signifikant (größere Häufigkeit von HLA-A10, A28, B13, B27, niedrigere von HLA-A2, Abwe-senheit von HLA-A11, B5, B14, B15, B17, B18, Bw21, Bw22, Bw35).

Bei der statistischen Bewertung aller kranken Kinder stellten wir eine signifikant höhere Häufigkeit von HLA-A28 (X2 = 5,69, p = 0,017, pc = 0,374) fest, jedoch nur vor der Durchführung der Korrektion des p-Wertes. Übrige Verände-rungen blieben nichtsignifikant: 
höhere Frequenz von HLA-B7, niedrigere von HLA-A2, Awl9, B5, B12, B13, das Nichtvorhandensein von HLA-B14, B17, B18, Bw21.

Diskussion

Unsere Resultate stimmen mit der Mehrheit der Studien iiber-ein, die keinen Zusammenhang zwischen den HLA-Antigenen und den Geschwülsten feststellen konnten. Statistisch signifikante Zusammenhänge zwischen HLA und Geschwülsten wurden nur ausnahmsweise nachgewiesen: Retinoblastom und HLA-Bw35 [3], Kolonkarzinom und HLA-B8 [5], Rektumkarzinom und HLA-Bw35 (in diesem Falle eine negative Be-ziehung) [5], Hypernephrom und HLA-Aw30/31 [9], Wilms-Tumor und HLA-A1 und/oder A9 [1, 11], Nasenrachenkarzi-nom und HLA-Bw46 (nur in chinesischer und mongoloider Population [13], Speiseröhrenkarzinom und HLA-B40 (bloß in türkisch-mongoloider Population) [5], Leberkrebs und HLA-A1 (bei Negern in Südafrika) [7], Aw24 bei Kranken mit Hoden-Teratomkarzinom und Metastasen [4]. Der Mechanismus einer Beziehung zwischen dem HLA-System und einer Erkrankung beruht auf zahlreichen Fakto-ren; aus diesen Gründen existiert bisher keine einfache Er-klärung dieser Erscheinung. Man nimmt an, daß sich hypo-thetische DS-Gene (disease-susceptibility genes die Gene für die Neigung zu einer Krankheit) am 6. Chromosom in der Nähe des HLA-Lokus befinden.

Die Ursache der so selten existierenden Verbindungen zwischen dem HLA-Lokus und den bösartigen Geschwülsten wird auf verschiedene Weise erläutert:

1. Größere Entfernung der DS-Gene vom HLA-Lokus als derGene für die Neigung zu anderen Krankheiten (z. B. zu anky-lotischer Spondylarthrose) [15].

Existenz eines Gleichgewichts zwischen den DS-Genen und HLA-Genen [15].

Existenz mehrerer Gene und äußerer Faktoren, die über die Entwicklung einer Geschwulst entscheiden [15].

Niedrige Zahl der untersuchten Kranken [5].

Literatur

Abrahámová, J.; Májsky, A.; Koutecky, J.: Wilmsuv nádor a HLA-Alokus. Cas.lék.ces. 776:1059-1061 (1977).

Bertrams, J.: HLA and disease association. Behring Inst. Mitt. 62: 69-92(1978).

Bertrams, J.; Schildberg, P.; Hopping, W.; Böhme, U., and Albert, E.: HLHL-A-A antigens in retinoblastoma. Tissue Antigens 3: 78-87(1973).

Carr, B. I. and Bach, F. H.: Possible association between HLA-Aw24 and metastatic testicular germ-cell tumours. Lancet 8130: 1346-1347 (1979).

Dausset, J.: HLA and association with malignancy. A critical view. HLA and Malignancy. A. R. Liss, New York (1977).

Hajdu, S. J. and Hajdu, E. O.: Malignant germ cell tumors. In: Cytopathology of sarcomas. W. B. Saunders, Philadelphia (1976).

Hammond, M. G; Appadov, B., and Brain, P.: HLA and cancer in South African Negroes. Tissue Antigens 9:1-7 (1977).

Knudson, A. G: Genetics and the etiology of childhood cancer. Pediat. Res. 10:513-517 (1976).

Kuntz, B. M. E.; Schmidt, G. D.; Scholz, S., and Albert, E. D.: HLA antigens and hypernephroma. Tissue Antigens 12: 407-408 (1978).

Májsky”, A.; Abrahámová, J.; Korinková, P., and Bek, V.: HLA system and testicular germinative tumors. Oncology 36: 228-231 (1979). 
Májsky”, A.; Abrahámová, J., and Koutecky, J.: Wilms’tumor and HLA antigens. Tissue Antigens 77:74 (1978).

Pierce, G B. and Dixon, F. J.: Testicular teratomas. Demonstration of teratogenesis by metamorphosis of multipotencial cells. Cancer 72:573-582 (1959).

Simmons, M. J.; Chan, S. H., and Day, N. E.: HLA and nasopharyngeal cancer. HLA and Malignancy, A. R. Liss, New York (1977).

Stevens, L. G.: Embryonic potency of embryoid bodies derived from transplantable testicular teratome of the mouse. Developmental Biol. 2: 285-297 (1960).

Terasaki, P. I.; Perdue, S. T., and Mickey, M. R.: HLA frequencies in cancer: A second study. Genetics of Human Cancer, Raven Press, New York (1977). 